Unfallchirurg 2012 $\cdot 115: 1044$

DOI 10.1007/s00113-012-2231-y

Online publiziert: 17. Juni 2012

(c) Springer-Verlag Berlin Heidelberg 2012
H. Twehues

Institut für medizinische Begutachtung Bielefeld

\title{
Ambulante Versorgung operativer Sprunggelenkfrakturen
}

\author{
Leserbrief zum Beitrag \\ Krätzig L, Gebhard F (2012) Ambulante \\ Versorgung operativer Sprunggelenk- \\ frakturen. Unfallchirurg 115:184
}

\section{Leserbrief}

\section{H. Twehues}

Institut für medizinische Begutachtung Bielefeld

Die Argumentation des Herrn Krätzig ist vordergründig und nur fadenscheinig.

Selbstverständlich kann von einem ausgebildeten Unfallchirurgen eine Sprunggelenkfraktur unter ambulanten Rahmenbedingungen sachgerecht korrekt im Sinne des Patienten operiert werden. Ebenso selbstverständlich setzt dies voraus, dass es sich um einen kooperativen Patienten handelt, der sich nach dem Eingriff nicht in seinen „Stall“ begibt.

Dass eine stationäre Behandlung Vorteile nach sich zieht, ist nicht zwangsläufig nachzuvollziehen, es darf hier nur in diesem Zusammenhang auf die Infektraten der Kliniken verwiesen werden.

Man würde im Übrigen auch den vielerorts ambulant tätigen hochqualifizierten Chirurgen und Orthopäden Unrecht tun, die zwischenzeitlich nahezu regelhaft ausgedehnte rekonstruktive Eingriffe im Bereich der Gelenke, z. B. Kniegelenke, durchführen, ohne dass hier es zu Komplikationen kommt, zumindest keine, die zahlenmäßig die in der Klinik überschreiten!

Hinzu kommt, dass es sich in der Regel bei den niedergelassenen Kollegen um entsprechende ausgebildete Fachärzte für Unfallchirurgie handelt, wobei nicht zwangsläufig vorausgesetzt werden kann, dass in der Klinik jeweils nach Facharztstandard operiert wird, hier müssen ja die entsprechenden Assistenten auch ausgebildet werden, wohlwissend, dass dies unter Anleitung eines Facharztes erfolgen soll.

Im Übrigen muss Herrn Krätzig dahingehend widersprochen werden, dass kein Facharzt für Unfallchirurgie im ambulanten Bereich ausgebildet worden ist.

Dieses ist naturgemäß unter Hinweis auf das Spektrum der ambulanten Möglichkeiten im vollen Umfang auch nicht möglich, niemand kommt auf die Idee, im ambulanten Rahmen den vollen unfallchirurgischen Standard bezüglich der großen Eingriffe zu gewährleisten.

Der Unterzeichner selbst hat zumindest für ein Jahr im Rahmen seiner niedergelassenen Tätigkeit die entsprechende Weiterbildungsermächtigung besessen, es kann davon ausgegangen werden, dass dies auch für andere Kollegen gilt.

Hier erfolgt offensichtlich die Diskussion aus der Interessenlage einer Klinik, medizinisch-wissenschaftlich lässt sich eine derartige Argumentation nicht aufrecht erhalten.

\section{Korrespondenzadresse}

\section{Dr. H. Twehues}

Institut für medizinische Begutachtung Bielefeld Feilenstraße 1, 33602 Bielefeld

imb-bielefeld@t-online.de

Interessenkonflikt. Der korrespondierende Autor gibt an, dass kein Interessenkonflikt besteht. 